\title{
Aggregation process of kaolinite clay
}

\author{
Letícia Morais ${ }^{1,{ }^{*}}$, Manoel Cordão-Neto ${ }^{1}$, and Alessandro Tarantino ${ }^{2}$ \\ ${ }^{1}$ University of Brasília, Department of Civil and Environmental Engineering, Brasília, Brasil \\ ${ }^{2}$ University of Strathclyde, Department of Civil and Environmental Engineering, Glasgow, UK
}

\begin{abstract}
Clay geomaterials pose a great challenge in geotechnical design due to their complex mechanical behaviour. Despite the vast research on clay mechanical behaviour, mechanisms occurring at the particle-scale still remain largely unknown. Particle-to-particle interactions include electro-chemical forces, which can be in turn associated with repulsive/attractive Coulomb interaction and attractive van der Waals force. This work aims to investigate the role of attractive forces (van der Waals and Coulomb) via their control of the process of aggregation (attractive forces tend to form aggregates of clay particles). Dry clay particles were compressed under high stress to reduce particles distances and activate attractive van der Waals and Coulomb forces. Particle size distribution was then measured using laser granulometry to explore aggregation formation. Laser granulometry tests were performed with and without ultrasound and with and without dispersant. Results show that the higher the compressive stress applied to the sample, the bigger is the 'particle' size measured by the laser granulometry, which corresponds to formation of aggregation due to attractive forces. Ultrasound appeared to disaggregate the aggregates thus suggesting that van der Waals and Coulomb forces are sensitive to dynamic loading.
\end{abstract}

\section{Introduction}

Mechanical and hydraulic behaviour of clays is controlled by processes occurring at the particle scale. Although great attention has been given to clay microstructure via SEM and MIP testing, the particle-scale origin of clay behaviour still remains largely unknown.

The main inter-particle forces between clay particles are van der Waals and Coulomb forces. In suspension Coulomb force is computed according to the Double Layer Theory $([1,2])$. [3] presented the first study about interparticle forces of cohesive soils, particularly double layer forces. [4] included the van der Waals forces in the study of clay soil. Experimental and numerical studies have been made since then with the aim to understand the role of interparticle forces on clay behaviour ([5-13]).

Coulomb force may be positive or negative. If charges surfaces have the same sign, they will be repulsive, otherwise they will be attractive. These forces will depend on the magnitude of the electrical field generated by charges of particle surface. In suspension double layer electrochemical forces occur due to the presence of unbalanced charges within in the clay particle. These charges create an electrical field that attracts ions forming a diffuse double layer. Particle surfaces with the same charge will repel each other and particle surfaces with opposite charge will attract each other. The double layer forces depend on the dielectric permittivity of the medium, temperature, valence of ions in solution and cation concentration in the free pore-water.
Van der Waals forces are long distance forces associated with the polarization of molecules. The asymmetric distribution of electron density generates a dipole moment within the molecule, which orients the molecule in a way that opposite charges stay side by side, thus generating attractive van der Waals forces. According to [14] these forces depend on the dielectric permittivity of the fluid.

Both van der Waals and Coulomb forces depend on the distance from the particle surface. At small distances, attractive van der Waals force tend to prevail with respect to Coulomb force if this force is repulsive. With increasing distance from the particle surface, Coulomb force dominate.

Understanding inter-particle forces is crucial to comprehend particle interactions. However, little attention has been devoted so far to attractive forces.

In this work, experimental tests were carried out to investigate clay particle attractive forces. The idea was to reduce particle distance by hyper-compaction and observe the final particle arrangement via laser granulometry tests.

According to the balance of interparticle forces, if inter-particle distances are small enough, van der Waals forces will be activated forming face-to-face aggregation. In this paper, another theory is put forward about face-toface attraction. It is shown that octahedral positively charged face can be attracted by tetrahedral negatively charged face again if inter-particle distances are small enough.

\footnotetext{
* Corresponding author: leticia.12.morais@.gmail.com
} 


\section{Background}

\subsection{Clay particle surface charge}

Charges on clay particle surfaces generate Coulomb interaction. The kaolinite particle has three surfaces that are electrically charged: the octahedral face, the tetrahedral face, and the edges. The presence of charges is mainly due to the permanent changes in the structure of the particle lattice, like isomorphous substitution, broken edges, and transient changes by protonation and deprotonation. The permanent changes always generate negative charges, but the transient changes may generate positive or negative charges in the particle surface.

There are two lines of thought about charge distribution around clay particles. The first approach is based on the point of zero charge (PZC), which for kaolinite is between $\mathrm{pH} 5$ and $6([9,15])$. Kaolinite particles are positively charged on the edges at low $\mathrm{pH}$ and negatively charged on the face. When in alkaline medium, kaolinite particles are negatively charged both on the edges and on the faces. Figure 1 shows kaolinite surface charges according to this line of research.

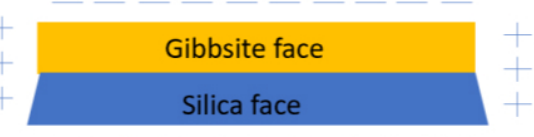

(a)



(b)

Fig. 1. Charge distribution around clay particle according to PZC (a) acid medium, (b) alkaline medium.

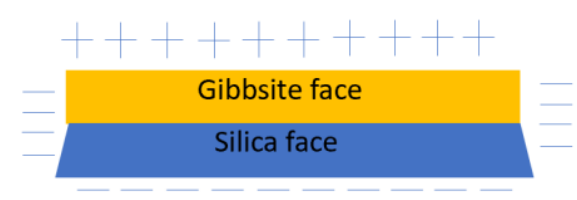

(a)

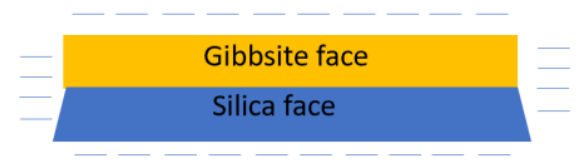

(b)

Fig. 2. Charge distribution around clay particle according to AFM (a) acid medium, (b) alkaline medium.

The second line of thought about surface charges is based on the measurements of the surface force using the Atomic Force Microscopy (AFM) ([16, 17]). They estimated the surface potential of the substrates of the faces (tetrahedral and octahedral) and edges of kaolinite particles for different $\mathrm{pH}$. The results present a different charge distribution around the clay particle. For acid medium the silica tetrahedral face will be negatively charged, and the alumina octahedral face will be positively charged. For alkaline medium both faces are negatively charged. The edges are negatively charged in acid and alkaline medium. Figure 2 presents charge distribution according to this line of research.

\subsection{Clay particle electric potential}

It is worth exploring the electrical field generated by the two surface charge models, PZC and AFM respectively. For the sake of simplicity, let us consider the electrical field generated by a clay particle equivalent to the electrical field generated by two circular disks uniformly charged.

For the case of the PZC surface charge model, the equivalent electrical layout is shown in Figure 3. The two circular disks are both negatively charged. The electrical potential on the axis $x$ of the disk generated by the lefthand disk and the right hand disk respectively are given by:

$$
\begin{gathered}
V_{\text {left }}=2 \pi \sigma_{P Z C} k\left[\sqrt{\left(x+\frac{d}{2}\right)^{2}+R^{2}}-\left|\left(x+\frac{d}{2}\right)\right|\right] \\
V_{\text {right }}=2 \pi \sigma_{P Z C} k\left[\sqrt{\left(x-\frac{d}{2}\right)^{2}+R^{2}}-\left|\left(x-\frac{d}{2}\right)\right|\right]
\end{gathered}
$$

where $\sigma_{\text {PZC }}$ is the surface charge in the PZC model, $k$ is the Coulomb constant $\left(k=9 \cdot 10^{9} \mathrm{~N} \mathrm{~m}^{2} \mathrm{C}^{-2}\right), R$ is the radius of the disk, $d$ is the spacing between the disks equal to the clay particle thickness.

The distribution of the electrical potential of the "clay particle' is obtained by superposing the electrical potential generated by the two disks. By assuming $R=0.5 \mu \mathrm{m}, d=0.1 \mu \mathrm{m}$, and $\sigma_{\mathrm{PZC}}=-0.5 \cdot \mathrm{mC} \cdot \mathrm{m}^{-2}$ (for a total net charge of $-1 \cdot \mathrm{mC} \cdot \mathrm{m}^{-2}$ ), the distribution shown in Figure 4 is obtained. Clearly, the electrical potential is always negative and is symmetric with respect to the particle mid-plane.



Fig. 3. Electrical layout for the PZC surface charge model 


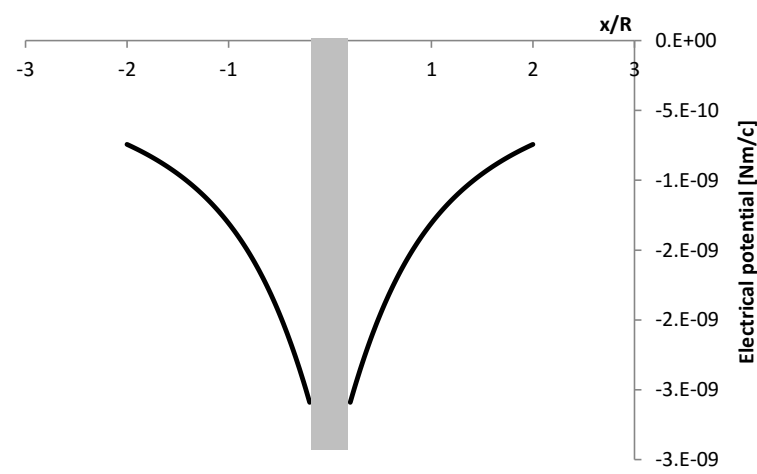

Fig. 4. Electrical potential for the PZC surface charge model

For the case of the AFM surface charge model, the equivalent electrical layout is shown in Figure 5. The two disks are now charged differently, the disk mimicking the octahedral face is charged positively and the disk mimicking the tetrahedral face is charged negatively. The electrical potential on the axis $x$ of the disk generated by the octahedral disk and the tetrahedral respectively are given by:

$$
\begin{aligned}
& V_{o c t}=2 \pi \sigma_{o c t} k\left[\sqrt{\left(x+\frac{d}{2}\right)^{2}+R^{2}}-\left|\left(x+\frac{d}{2}\right)\right|\right] \\
& V_{t e t}=2 \pi \sigma_{t e t} k\left[\sqrt{\left(x-\frac{d}{2}\right)^{2}+R^{2}}-\left|\left(x-\frac{d}{2}\right)\right|\right]
\end{aligned}
$$

where $\sigma_{\text {oct }}$ is the surface charge of the octahedral face and $\sigma_{\text {tet }}$ is the surface charge of the tetrahedral face.



Fig. 5. Electrical layout for the AFM surface charge model

By assuming $R=1 \mu \mathrm{m}, d=0.2 \mu \mathrm{m}, \sigma_{\text {oct }}=+5 \cdot \mathrm{mC} \cdot \mathrm{m}^{-2}$ and $\sigma_{\text {tet }}=-6 \cdot m C \cdot m^{-2}$ (for a total net charge of $-1 \cdot \mathrm{mC} \cdot \mathrm{m}^{-2}$ ), the distribution of the electrical potential shown in Figure 6 is obtained. It is worth noticing that the two surface charge models, the PZC and the AFM respectively, are here compared by assuming the same total net charge of $1 \cdot \mathrm{mC} \cdot \mathrm{m}^{-2}$. The values chosen for $\sigma_{\text {oct }}$ and $\sigma_{\text {tet }}$ are consistent with the measurement presented by [16].

The electrical potential is no longer symmetric with respect to the particle mid-plane. Because the total net charge is negative, the electrical potential is generally negative with the exception of the region close to the octahedral face where the electrical potential becomes positive. Figure 6 therefore shows that face-to-face double layer attractive forces can be activated if an octahedral face front on to a tetrahedral face and if the two particles are brought sufficiently closer to each other.

It would therefore appear that face-to-face attraction is contemplated by the AFM model. Similarly to van der Waals attraction, closer inter-particle spacing would be required to activate double-layer face-to-face attraction.

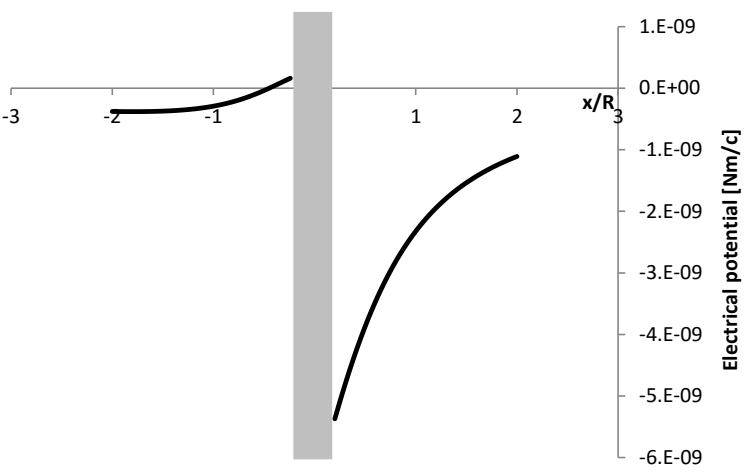

Fig. 6. Electrical potential for the AFM surface charge model

\subsection{Particle interaction and microstructural behaviour of clays}

The presence of charges around clay particles will generate attractive or repulsive interactions. Arrangements of clay particles may include face-to-face, face-to edge, edge-to-edge, and disperse (Figure 7).

The pore-fluid properties will influence in the charge distribution around the particle. The distance between particles will dictate which force will prevail (van der Waals or Coulomb). Both aspects are important to understand particle arrangement and which force is being activated.

The existence of two lines of research about charge distribution will generate some hypothesis about particle interactions if a sample of kaolinite is compacted under high loads. Considering that particles will be aligned faceto-face, particle interactions may occur only by van der Waals forces or by both van der Waals and Coulomb forces.

Studies $([11,13])$ were done to observe the behaviour of compressed clay. [11] carried out consolidation of kaolinite at low and high $\mathrm{pH}$. It was observed that low $\mathrm{pH}$ kaolinite presents an initial higher volume than high $\mathrm{pH}$, suggesting that edge-to-face interaction occur between particles. With loading both samples tend to equal final volume. [13] carried out consolidation tests with kaolinite with different pore fluid (water, acetone and air). They observed that the higher the dielectric permittivity of the 
fluid, the lower is the void ratio of the sample. This suggests that the repulsive Coulomb force is lower at high dielectric permittivity.

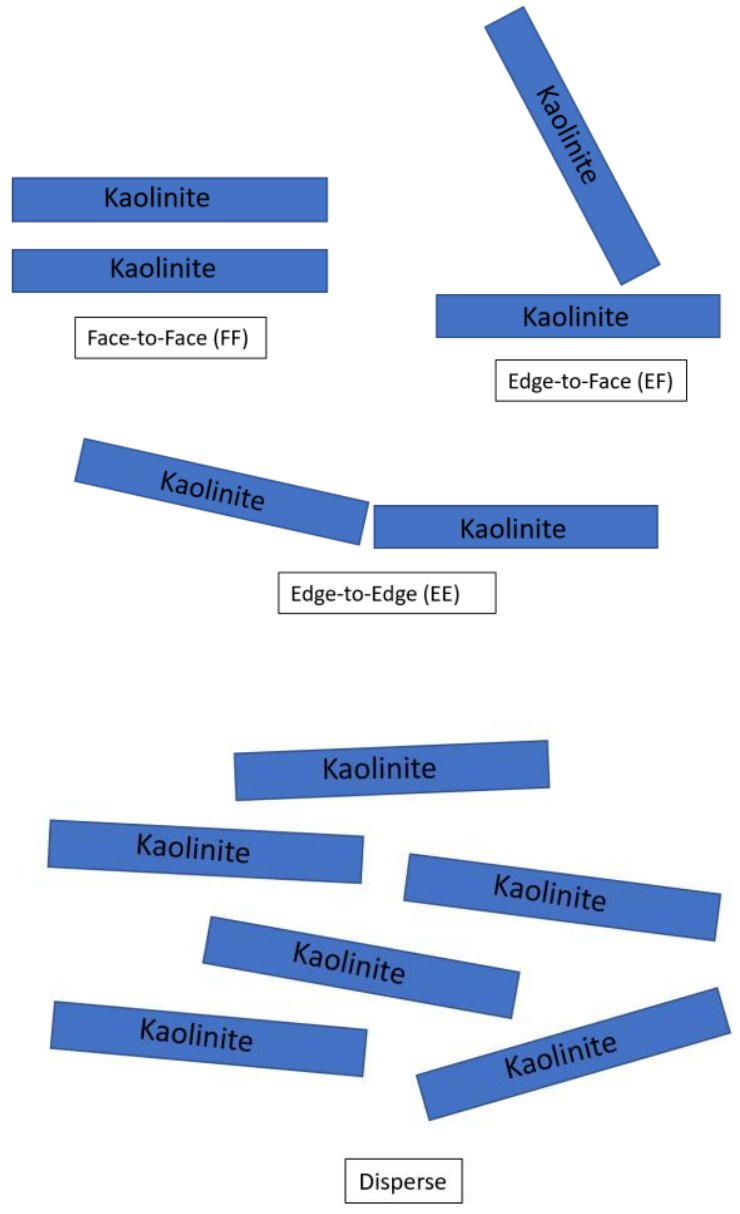

Fig. 7. Arrangements of clay particles.

\section{Experimental investigation}

\subsection{Material}

The material used in the tests was a Kaolinite with particle density of $2,60 \mathrm{~g} / \mathrm{cm}^{3}$, liquid limit of $49 \%$ and plasticity limit of $41 \%$. The material is composed of $43,3 \%$ of $\mathrm{SiO}_{2}$ and $39,9 \%$ of $\mathrm{Al}_{2} \mathrm{O}_{3}$.

\subsection{Tests performed}

The study included compaction tests and laser granulometry tests. Kaolin powder at its hygroscopic water contents was statically compacted to $2 \mathrm{MPa}, 10 \mathrm{MPa}$, $50 \mathrm{MPa}$ and $100 \mathrm{MPa}$ vertical stress. This process tended to decrease particles distances possibly favouring the process of particle aggregation due to the activating of attractive Coulomb and attractive van der Waals forces. The load was maintained for 15 minutes in order to stabilize particle arrangement. Figure 8 shows a sample of compacted kaolinite.

The compacted sample were then smashed and left $24 \mathrm{~h}$ in distilled water or in a solution of water with the addition of hexametaphosphate. No stirring was applied before the tests. Finally, the compacted samples were subjected to laser granulometry test to obtain 'particle' size distribution. The tests were performed with and without the use of ultrasound. The hexametaphosphate is a known dispersant which neutralizes positive surface charges of kaolinite, leaving all faces with negative charges. The ultrasound promotes a dynamic stirring in the samples, tending to disaggregate 'particles'.

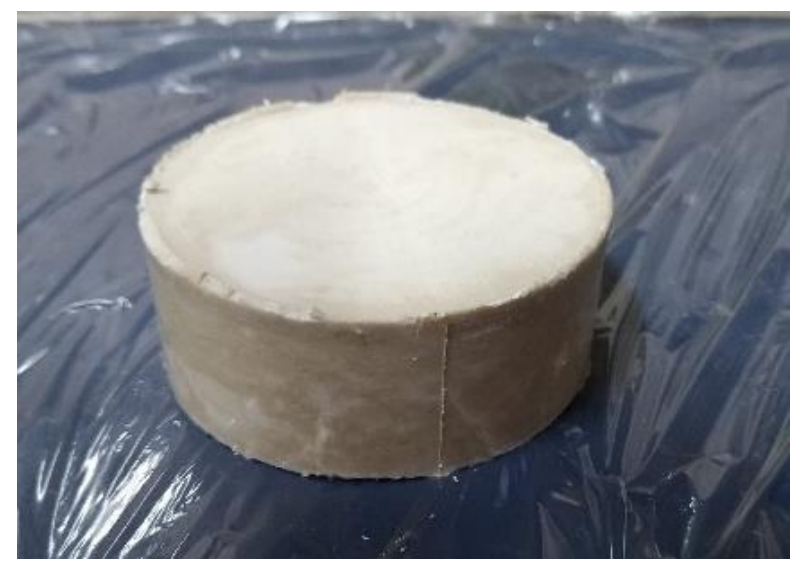

Fig. 8. Compacted sample

\section{Results and discussion}

Figures 9 and 10 show the 'particle' size distribution obtained with laser granulometry tests, using water with the addition of hexametaphosphate and without the use of ultrasound. With increasing compaction vertical stress, it is possible to observe that the size of the aggregation increases (the 'particle' size distribution shifts to the right). These results therefore show that an aggregation process tends to occur between clay particles with increasing loading.

Figure 10 also shows that aggregation is initially bimodal and tends to become unimodal with increasing loading. The results indicate that attractive double layer and/or van der Waals forces were likely activated by decreasing inter-particle distance.



Fig. 9. Curves of samples subjected to laser granulometry tests with hexametaphosphate and without ultrasound. 


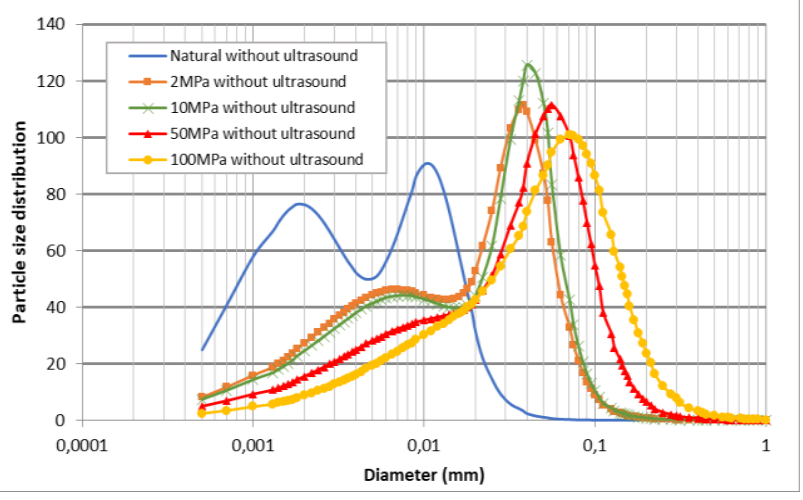

Fig. 10. Curves of density of samples subjected to laser granulometry tests with hexametaphosphate and without ultrasound

Figure 11 shows the particle distribution of samples subject to laser granulometry test with the use of hexametaphosphate and ultrasound. The curves of loaded samples are overlapping the curve of the natural samples, indicating that the aggregation formed by the compaction was destroyed by the ultrasound.

Finally, tests of laser granulometry in distilled water without the addition of hexametaphosphate were performed. The aim of the test is to evaluate if the hexametaphosphate was interfering with the process of aggregation. Figure 12 shows the results of the tests with and without hexametaphosphate. Hexametaphosphate did not disaggregate the 'particles' since results are the same as the tests using distilled water only.

The dispersant has a high $\mathrm{pH}$, which would generate a negative surface charge at all faces of kaolinite. As the hexametaphosphate (dispersant) did not interfere with the process of aggregation, it can be inferred that aggregation was not associated with the formation of 'floccules', which would have been destroyed by the change of particle surface charge from positive to negative. Aggregation was likely genuinely associated with face-toface aggregation due to attractive Coulomb and/or van der Waals forces.

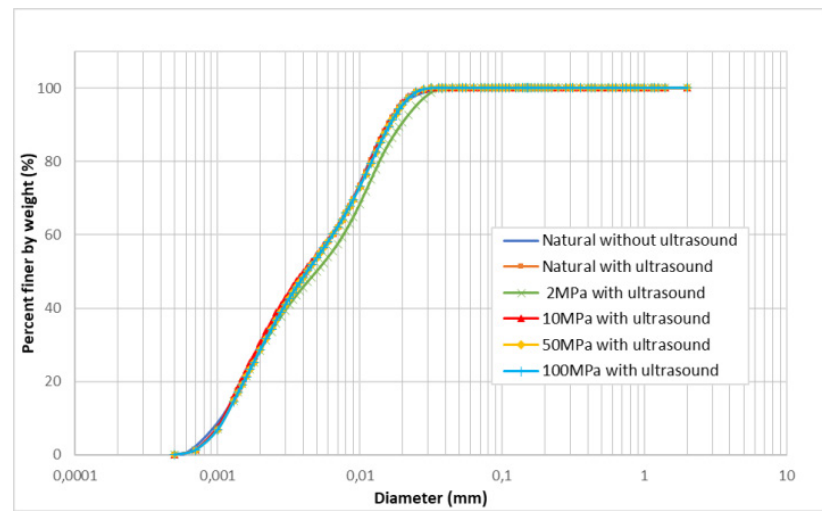

Fig. 11. Curves of samples subjected to laser granulometry tests with hexametaphosphate and with ultrasound.

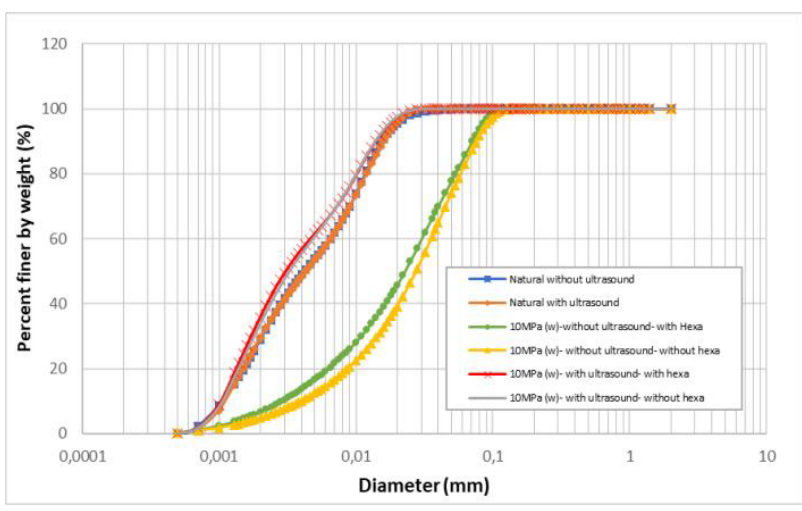

Fig. 12. Curves of samples subjected to laser granulometry tests with hexametaphosphate and distilled water and with and without ultrasound.

\section{Conclusions}

This work explored the process of aggregation of kaolinite particles due to compaction under high stresses. Attractive forces were expected to be activated between clay particles.

In order to understand particle interaction and the nature of those forces, laser granulometry tests were performed. These tests showed that the higher the compaction stress the larger is the size of the aggregates. Also, it was observed that the aggregates were stable in water and hexametaphosphate, but they were not stable when dynamic stirring was applied via ultrasound.

Aggregations are formed due to face-to-face attraction (Fig. 13) rather than edge-to-face flocculation since no differences were observed in the laser granulometry tests carried out with and without the addition of the dispersant (hexametaphosphate).

At this stage, it not possible to speculate if aggregation is mainly due to van der Waals force, attractive Coulomb force or both. Theoretically, these forces both activate at relatively small inter-particle distances.

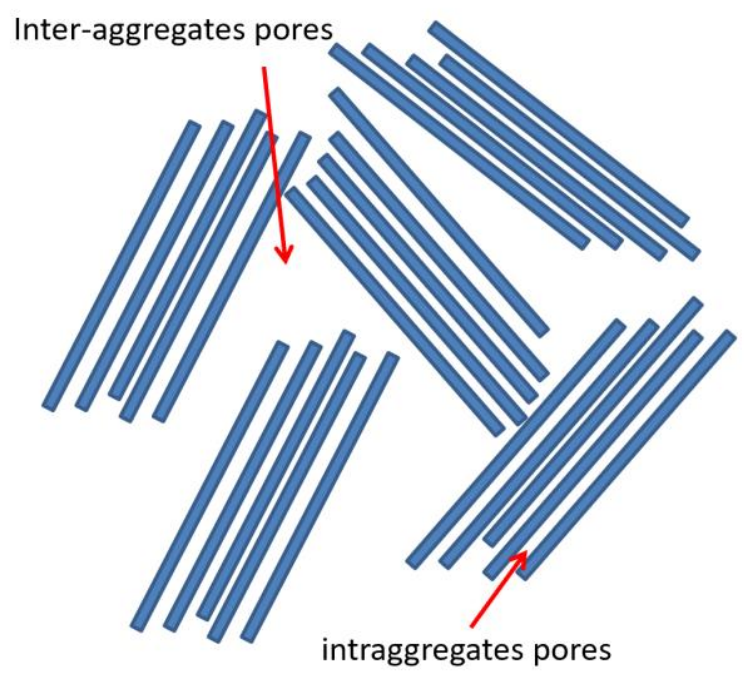

Fig. 13. Curves of samples subjected to laser granulometry tests with hexametaphosphate and distilled water and with and without ultrasound. 
The authors acknowledge the support provided by the European Commission by Marie Curie IRSES project GREAT (FP7PEOPLE-2013-IRSES-612665) and by the "Conselho Nacional de Desenvolvimento Científico e Tecnológico" CNPq of Brazil.

\section{References}

1. B. Derjaguin, L. Landau, Acta Physicochim, 14, 633$662(1941)$

2. E. J. W., Verwey, J.T.Overbeek, Theory of stability of lyophobic colloids, New York: Elsevier, (1948)

3. G. H. Bolt, Geotechnique, 6, 86-93 (1956)

4. C. A. Moore, J.K. Mitchel, J. Geotechnique, 24, 627640 (1974)

5. A. Anandarajah, J. Geotech. Engrg, 120, 1593-1613 (1994)

6. A. Anandarajah, J. Chen, Soils and foundations, 37, 27-37 (1997)

7. J. Chen, A. Anandarajah, H. Inyang, J. Geotechnical and Geoenvironmental Engineering, 126, 798-807 (2000).

8. M. Yao, A. Anandarajah,J. Engineering Mechanics, 129, 585-596 (2003)

9. Y. H. Wang, W.K. Siu, Canandian Geotechnical Journal, 43 (2006)

10. M. Pedrotti, A. Tarantino, Unsaturated Soils: Research \&Applications , 739-745 (2014)

11. B. C. F. L. Lopes, PhD Thesis, University of Brasília (2016)

12. A. G. Pagano, A. Tarantino, M. Pedrotti, V. Magnanimo, K. Windows-Yule, T. Weinhart, Powders \& Grain, 140, (2017)

13. M. Pedrotti, A. Tarantino, Geotechnique, 68, 666683 (2018)

14. J. Mitchel, K. Soga, Fundamentals of Soil Behaviour, Jon Wiley and Sons Inc. Hoboken, NJ (2005)

15. V. Gupta, M. A. Hampton, J. R. Stokes, A. V. Nguyen, J. D. Miller, J. Colloid Interface Sci., 359, 95-103 (2011)

16. V. Gupta, J.D. Miller, J. Colloid Interface Sci., 344, 362-371 (2010)

17. J. Liu, L. Sandaklie-Nikolova, X. Wang, J.D. Miller, J. Colloid Interface Sci, 420, 35-40 (2014) 